ISSN-PRINT 1794-9831 / E-ISSN 2322-7028

Vol. 18 No 3 / sep - dic, 2021 / Cúcuta, Colombia.

doi https://doi.org/10.22463/17949831.3060

Original

\title{
Carga del cuidador y resiliencia de enfermeros en atención domiciliaria durante pandemia covid-19
}

\section{Carga do cuidador e resiliência de enfermeiros em cuidados domiciliares durante a pan- demia covid-19}

\section{Caregiver burden and resilience of nurses in home care during a covid-19 pandemic}

\author{
Ángela María Henao-Castaño ${ }^{1^{*}}$ \\ Jeimmy Johana Blanco-Caviedes ${ }^{2}$ \\ Elizabeth Fajardo-Ramos ${ }^{3}$
}

\section{Resumen}

La necesidad de servicios de atención domiciliaria, especialmente para adultos mayores va en aumento, y en época de pandemia hace que en los cuidadores formales aumente su carga como cuidadores. Objetivo: Describir la carga del cuidador y la resiliencia de los enfermeros que brindan atención domiciliaria en época de pandemia. Métodos: Estudio cuantitativo, descriptivo y transversal. Se realizó en una Institución prestadora de salud de atención domiciliaria en el mes de noviembre del 2020. La población estuvo conformada por 20 enfermeros. El muestreo fue no probabilístico por conveniencia. Se utilizó la Escala de resiliencia de Wagnild, \& Young y la carga de cuidador de Zarit. Para el análisis de los datos se empleó IBM SPSS Statistics 22, obteniendo la estadística descriptiva e inferencial relacionada con: medias, desviaciones estándar, frecuencias, porcentajes y prueba de Fisher. Resultados: El $94 \%$ de los cuidadores formales eran mujeres. Se evidencio una carga intensa del $20 \%$ de los cuidadores; del mismo modo se observó un bajo nivel de resiliencia en cuidadores que viven con sus hermanos frente a un alto nivel de resiliencia en cuidadores que viven solos, independientes. Conclusión: En épocas de pandemia las responsabilidades laborales en la atención domiciliaria, especialmente con adultos mayores aumentan, así como las amenazas del medio, los aspectos personales del cuidador formal y el número de personas a cargo, son factores que hacen que en esta época las condiciones laborales y personales se tornen difíciles, ocasionando una fuerte carga para el cuidador.

Palabras clave: Atención de Enfermería; resiliencia psicológica; infecciones por coronavirus; atención domiciliaria; cuidador.

\section{Autor de correspondencia*}

1* Doctora en enfermería. Magister en Educación. Profesora Asociado. Facultad de Enfermería Universidad Nacional de Colombia. Bogotá, Colombia. Grupo Cuidar SAS. Correo: angmhenaocas@unal.edu.co (iD) 0000-00034203-0016

2 Psicóloga. Magister en neuropsicología y educación. Grupo Cuidar SAS. Correo: jeimmyblancocaviedes@ gmail.com (D) 0000-0003-2146-9281

${ }^{3}$ Enfermera, Magister en Enfermería. Profesor Titular. Universidad del Tolima. Correo: efajardo@ut.edu.co (D) 0000-0002-3484-1620

\footnotetext{
Abstract

The need for home care services, especially for older adults, is increasing, and in times of pandemic, formal caregivers are used to support this care, leading them to present a caregiver burden. Objetive: Describe the caregiver burden and resilience of nurses who provide home care in times of pandemic. Methods: Quantitative, descriptive, cross-sectional study, carried out in an Institution that provides home care health in the month of November 2020. The population was made up of 20 nurses. The sampling was non-probabilistic for convenience. The Zarit Resilience and Caregiver Burden Scale was used. For data analysis, IBM SPSS Statistics 22 was used, from which descriptive and inferential statistics were obtained, such as means, standard deviations, frequencies, percentages and Fisher's test. Results: $95 \%$ of the formal caregivers were women, an intense burden was evidenced in $20 \%$ of the caregivers
}

Recibido: 22 junio 2021

Aprobado: 26 agosto 2021

\section{Para citar este artículo}

Henao-Castaño AM, Blanco-Cavides JJ, Fajardo-Ramos E. Carga del cuidador y resiliencia de enfermeros en atención domiciliaria durante pandemia covid-19. Rev. cienc. cuidad. 2021; 18(3):64-73. https:// doi.org/10.22463/17949831.3060

(C) Universidad Francisco de Paula Santander. Este es un artículo bajo la licencia CC-BY-NC-ND 


\section{Scientific Journal of Nursing}

and a low level of resilience in those caregivers living with a number of siblings and a high level of resilience in caregivers who live alone, independent. Conclusion: In times of pandemic, work responsibilities in home care, especially of older adults, increase, threats from the environment, and personal aspects such as the number of people in charge make work and personal conditions difficult to carry and carry at this time. in charge of the caregiver.

Keywords: Nursing care, psychological resilience, coronavirus infections, home care, caregiver.

\section{Resumo}

A necessidade de serviços de atenção domiciliar, principalmente para idosos, está aumentando e, em tempos de pandemia, cuidadores formais são utilizados para apoiar esse cuidado, levando-os a representar uma sobrecarga de cuidador. Objetivo. Descreva a carga do cuidador e a resiliência dos enfermeiros que prestam atendimento domiciliar em tempos de pandemia. Materiais e métodos. Estudo quantitativo, descritivo, transversal, realizado em uma Instituição que presta atenção à saúde no domicílio no mês de novembro de 2020. A população foi composta por 20 enfermeiras. A amostragem foi não probabilística por conveniência. Foi utilizada a Escala de Resiliência e Sobrecarga do Cuidador de Zarit. Para a análise dos dados, foi utilizado o IBM SPSS Statistics 22, a partir do qual foram obtidas estatísticas descritivas e inferenciais, como médias, desvios-padrão, frequências, percentuais e teste de Fisher. Resultados. 95\% dos cuidadores formais eram mulheres, foi evidenciada sobrecarga intensa em $20 \%$ dos cuidadores e baixo nível de resiliência naqueles cuidadores que vivem com vários irmãos e alto nível de resiliência nos cuidadores que vivem sozinhos, independentes. Conclusão. Em tempos de pandemia, aumentam as responsabilidades laborais no atendimento domiciliar, principalmente dos idosos, ameaças do meio ambiente e aspectos pessoais, como o número de responsáveis, dificultam o trabalho e as condições pessoais neste momento. responsável pelo cuidador.

Palavras-chave: Cuidado de enfermagem, resiliência psicológica, infecções por coronavírus, atenção domiciliar, cuidador.

\section{Introducción}

En los últimos años ha adquirido importancia la atención domiciliaria, referida especialmente con las actividades en el servicio de enfermería, convirtiéndose en un alivio para el sistema de salud en Colombia y para aquellas familias que tienen un ser querido padeciendo una enfermedad crónica y degenerativa. En este contexto el rol del enfermero juega un papel primordial en todos los procesos de apoyo y acompañamiento en la rehabilitación y sostenimiento de las funciones básicas.

El cuidado de pacientes crónicos para el personal de enfermería domiciliario implica diferentes cargas y situaciones a las que debe someterse afectando directamente la salud mental y física del enfermero. Además, dichas situaciones se presentan con mayor frecuencia cuando en el núcleo familiar del paciente no existe un cuidador que apoye las actividades diarias, debido a esto no solamente se enfrentan a las dificultades como profesionales, sino que además se ocupan de la carga del familiar. En un estudio realizado respecto a sobrecarga, empatía y resiliencia en cuidadores de personas dependientes se describe la importancia de tener el apoyo de un profesional en el domicilio, ya que este disminuye la sobrecarga del cuidador primario y la demanda psicosocial se comparte (1).

Por resiliencia se entiende como la capacidad que tiene un individuo de desarrollarse exitosamente a pesar de enfrentarse a situaciones desfavorables, estresantes o desafiantes (2). También, se la conceptualiza como una competencia adaptativa gracias a la cual un sujeto puede responder con patrones positivos a amenazas reales o factores de riesgo biológicos, individuales, interpersonales, sociales y/o comunitarios (3). 
Este concepto sobre resiliencia relaciona las cualidades psicológicas positivas del sujeto con los factores protectores de su contexto a favor de la construcción favorable de los procesos de afrontamiento, recuperación, transformación y enriquecimiento individual tras experiencias difíciles e indeseables (4).

Por otra parte, existen diversos instrumentos de evaluación de la resiliencia (5), así como de su utilidad en personal de salud y enfermeros (6). Entre ellos se encuentra la Resilience Scale (RS-25) desarrollada por Wagnild y Young (7) que permite identificar el grado de resiliencia psicológica; además, posee buenas propiedades psicométricas en cuanto a su fiabilidad y validez en su versión original y en español (8).

Las potencialidades de la resiliencia en los profesionales de la enfermería se las debe tener muy en cuenta a la hora de diseñar programas, pero que además cuenten con una relación interdisciplinaria, para fomentar la atención asertiva y disminuir las amenazas del medio en que se desarrollan las actividades de la enfermería ya sean intramurales y extramurales (9). Los componentes cognitivos y emocionales en profesionales de la salud son factores influyentes en la organización del trabajo, así como las condiciones de trabajo (10).

Simultáneamente, la carga del cuidador formal hace referencia a las demandas necesarias para cuidar a los adultos mayores dependientes, con un nivel de competencia y responsabilidad dentro del contexto de estrés percibido (11). Así, el sentimiento de carga del cuidador se ha revelado como un importante factor tanto en la utilización de servicios de larga estancia como en la calidad de vida de los cuidadores.

Hay que mencionar además, que el instrumento de evaluación de mayor uso en el idioma español es la Entrevista de Percepción de Carga del Cuidado de Zarit (12). Se trata de un cuestionario que surgió para la evaluación de la carga de cuidadores de personas con demencia; del mismo modo, determina la carga que experimenta el cuidador mediante una puntuación global, presentando así una concepción unidimensional a pesar de contener ítems que se refieren a distintos aspectos de la carga (13). Se debe precisar, que la carga del cuidador no se limita solamente a los cuidadores informales (14), lo que ha permitido que el instrumento mida la carga del cuidador formal en el personal de enfermería en atención directa (15).

Se debe precisar que estos profesionales de la salud tienen la capacidad de manejar diferentes situaciones bajo mucha presión, destacándose su capacidad para adaptarse con éxito. Así mismo, se centran en el abor- daje de factores individuales que protegen los efectos del estrés prolongado (16), facilitándoles una mayor capacidad de relacionarse y reconocer la necesidad de atención que tienen otras personas (17).

En cuanto a las dificultades que todo el personal de salud en tiempos de pandemia ha tenido que afrontar se encuentran: la discriminación, la angustia de tener que salir a cumplir con sus labores diarias y poner en riesgo no solamente su salud, si no la de su núcleo familiar, a las que se suman todos los protocolos que deben cumplir para prestar un servicio con calidad humana.

Conviene subrayar, que los diferentes aspectos psicosociales que ha tenido que enfrentar el personal de la salud en las diferentes unidades es reportado en un estudio sobre la vulnerabilidad y resiliencia de las cuidadoras familiares en medio de la pandemia de la Covid-19, en donde se concluye que la capacidad que tienen los profesionales y cuidadores para asistir al personal que los necesita ha demostrado su capacidad para dejar a un lado todo tipo de creencias fortaleciendo las políticas sociales y de salud (18).

En tiempos de pandemia la población más afectada son los adultos mayores con enfermedades de base, razón por la cual son los principales beneficiarios del servicio de enfermería domiciliaria. En consecuencia, los profesionales deben participar activamente en el cumplimiento de protocolos y estrategias que ayuden a mantener a salvo a su paciente y familia, lo que ocasiona una carga adicional a los quehaceres diarios. Por tanto, una de las recomendaciones en tiempos de COVID-19 respecto a la atención domiciliaria es que los enfermeros evalúen y comprueben si el entorno del hogar es adecuado para la continuidad de la atención; pero además examinar si el paciente y la familia están en condiciones de cumplir con las precauciones recomendadas como parte del aislamiento (19).

Actualmente los servicios de atención domiciliaria, especialmente los de enfermería se han centrado en mantener a sus pacientes de alta vulnerabilidad alejados de los posibles focos de contagio; también, se han extendido a pacientes POS- COVID, con el fin de rehabilitarlos y acompañarlos para que retornen a su vida laboral y social.

Por su parte, la comunidad científica ha destacado la labor que cumplen los profesionales que se encuentran en primera línea. Igualmente, ha enfatizado sobre la capacidad de los enfermeros domiciliarios para superar todas las problemáticas que deben afrontar, con el objeto de brindar un servicio con calidad humana. 
Hay que mencionar una publicación de atención primaria y domiciliaria al final de la vida en tiempos de COVID-19, en donde se subraya sobre la importancia de la experiencia adquirida por los profesionales de atención domiciliaria para brindar confort y ofrecer una visión integral e integradora en el manejo y cuidado de estos pacientes (20). En función de lo planteado anteriormente se deduce que la responsabilidad de los profesionales en atención domiciliaria no solo demuestra su capacidad de adaptación a diferentes situaciones, sino que también están capacitados para afrontar las cargas en diferentes momentos y cómo estas afectan su entorno profesional y laboral $(21,22)$.

En consecuencia es conveniente realizar un estudio sobre lo que significa la carga del cuidador y su resiliencia, especialmente en tiempos de pandemia, que ayude a entender las dificultades que deben afrontar estos profesionales de la salud.

\section{Objetivos}

\section{Objetivo general}

Determinar la sobrecarga del cuidador y nivel de resiliencia en enfermeros de atención domiciliaria en época de pandemia

\section{Materiales y Métodos}

Es un estudio con enfoque cuantitativo, descriptivo y transversal. La muestra fue no probabilística por conveniencia y estuvo conformada por 20 enfermeros que laboran en una Institución prestadora de salud domiciliaria. Los participantes contaban con una experiencia laboral mayor de un año en la institución y brindaron cuidado en el domicilio durante la pandemia. Los participantes fueron convocados por correo electrónico y posteriormente se agendó el horario de la participación, que se efectuó en el mes noviembre del 2020, en las instalaciones de la institución, de manera individual.

Para la recolección de la información se contó con una ficha desarrollada por las investigadoras quienes recolectaron los datos sociodemográficos; de igual manera, se utilizó la Escala de Resiliencia de Wagnild, \& Young validada al español por Heilemann, la cual se aplicó en una muestra conformada por 145 mujeres mexicanas cuyo idioma nativo fue el español. El instrumento cuenta con 25 ítems agrupados en 2 dimensiones: Competencia personal y Aceptación de uno mismo y de la vida. La fiabilidad se estimó con alfa de Cronbach (alfa $=0,93)$; por otra parte, la validez del constructo se demostró por una correlación positiva significativa entre la resiliencia y la satisfacción con la vida $(\mathrm{r}=0.36 ; \mathrm{p}<0.001)$, y una correlación negativa significativa entre la resiliencia y los síntomas depresivos $(r=-0.29 ; p<0.01)(23)$.

Como complemento, se empleó el cuestionario de sobrecarga del cuidador de Zarit, instrumento que cuantifica el grado de sobrecarga que padecen los cuidadores de las personas dependientes. Consta de un listado de 22 afirmaciones que describen cómo se sienten a veces los cuidadores; frente a cada aserción, el cuidador debe indicar la frecuencia con que se siente así, utilizando una escala que consta de: 0 (nunca), 1 (rara vez), 2 (algunas veces), 3 (bastantes veces) y 4 (casi siempre). Cuenta con una sensibilidad que oscila entre un $36,84 \%$ y $81,58 \%$, una especificidad entre $95,99 \%$ y $100 \%$, un valor predictivo positivo entre $71,05 \%$ y $100 \%$ y valores predictivos negativos entre el $91,64 \%$ y el $97,42 \%(24)$.

Para el análisis de la información, los datos se organizaron en una plantilla Microsoft Excel y se procesaron en el software SPSS 22 utilizando análisis univariado y bivariado.

En cuanto a las consideraciones éticas, los participantes conocieron los objetivos del estudio y posteriormente dieron su consentimiento informado. Se debe agregar que el presente estudio tuvo en cuenta los lineamientos para la investigación biomédica atendiendo al artículo 11, capítulo 1 de la Resolución 8430 de 1993, donde se establecen las normas científicas, técnicas y administrativas para la investigación en salud en Colombia. Del mismo modo se obtuvo el aval número 18420 del Comité de Ética la institución de atención domiciliaria (25).

\section{Resultados}

El número de participantes estuvo conformado por 20 enfermeros que ejercían labores de cuidado en atención domiciliaria a pacientes crónicos, durante la pandemia en una institución prestadora de salud de la ciudad de Ibagué. Dentro de las características generales de los participantes se pueden mencionar: 19 eran mujeres y 1 hombre, con edades comprendidas entre los 22 y 50 años. En cuanto al estado civil se observó un $40 \%$ de solteros que laboran en hospitalización domiciliaria, trabajando más del $100 \%$ por año.

En la Tabla 1 se registran las características sociodemográficas, la carga del cuidador con sobrecarga y sobrecarga intensa en $25 \%$. Sus edades oscilan entre los 20 y 40 años y nivel socioeconómico medio. 


\section{Scientific Journal of Nursing}

Tabla 1. Carga de cuidador según variables sociodemográficas

\begin{tabular}{|c|c|c|c|c|c|}
\hline Variables & No sobrecarga $(n=15)$ & Sobrecarga $(n=1)$ & $\begin{array}{l}\text { Sobrecarga in- } \\
\text { tensa }(n=4)\end{array}$ & Total & valor $p^{*}$ \\
\hline \multicolumn{6}{|l|}{ Genero } \\
\hline Femenino & 14 & 1 & 4 & 19 & \multirow[t]{2}{*}{1.00} \\
\hline Masculino & 1 & 0 & 0 & 1 & \\
\hline \multicolumn{6}{|l|}{ Edad } \\
\hline 20 - 25 años & 1 & 0 & 2 & 3 & \multirow[t]{6}{*}{0,411} \\
\hline 26 - 30 años & 5 & 0 & 1 & 6 & \\
\hline 31 - 35 años & 1 & 0 & 0 & 1 & \\
\hline 36 - 40 años & 2 & 1 & 1 & 4 & \\
\hline 41 - 45 años & 4 & 0 & 0 & 4 & \\
\hline 46 - 50 años & 2 & 0 & 0 & 2 & \\
\hline \multicolumn{6}{|l|}{ Vive con } \\
\hline Familiares & 2 & 0 & 1 & 3 & \multirow[t]{4}{*}{0,287} \\
\hline Independiente & 3 & 0 & 0 & 3 & \\
\hline Otro & 6 & 1 & 0 & 7 & \\
\hline Padres & 4 & 0 & 3 & 7 & \\
\hline \multicolumn{6}{|l|}{$\mathbf{N}^{\circ}$ hermanos } \\
\hline 1 hermano & 3 & 0 & 0 & 3 & \multirow[t]{5}{*}{0,032} \\
\hline 2 Hermanos & 1 & 0 & 1 & 2 & \\
\hline 3 Hermanos & 6 & 1 & 0 & 7 & \\
\hline 4 Hermanos & 4 & 0 & 0 & 4 & \\
\hline No Aplica & 1 & 0 & 3 & 4 & \\
\hline \multicolumn{6}{|c|}{ Nivel socioeconómico } \\
\hline Bajo & 1 & 0 & 0 & 1 & \multirow[t]{3}{*}{0,718} \\
\hline Medio & 10 & 1 & 4 & 15 & \\
\hline Medio Bajo & 4 & 0 & 0 & 4 & \\
\hline
\end{tabular}

* prueba exacta de Fisher

Fuente. Elaboración propia resultados estudio

Se puede observar que, según la escala de carga de Zarit, el cuidador con sobrecarga intensa es aquel con un menor número de hermanos (2 hermanos) tiende a vivir con los padres o familiares, aunque no hay una asociación muy fuerte; mientras que los que no presentan sobrecarga tienen más hermanos, pero viven de manera independiente o con otras personas, además tienen muy baja ansiedad.

En la Tabla 2, se presentan los resultados de resiliencia y las variables sociodemográficas. Al respecto no se observaron diferencias estadísticamente significativas entre aquellos que tenían bajo o moderado nivel de resiliencia y los que presentaron alta resiliencia. 
Scientific Journal of Nursing

Tabla 2. Resiliencia según variables sociodemográficas

\begin{tabular}{|c|c|c|c|c|}
\hline Variables & Bajo/moderado $(n=15)$ & Alta $(n=5)$ & Total & valor $\mathrm{p}^{*}$ \\
\hline \multicolumn{5}{|l|}{ Genero } \\
\hline Femenino & 14 & 19 & 19 & \multirow[t]{2}{*}{0,75} \\
\hline Masculino & 1 & 1 & 1 & \\
\hline \multicolumn{5}{|l|}{ Edad } \\
\hline 20 - 25 años & 3 & 0 & 3 & \multirow[t]{6}{*}{0,42} \\
\hline 26 - 30 años & 5 & 1 & 6 & \\
\hline 31 - 35 años & 0 & 1 & 1 & \\
\hline 36 - 40 años & 2 & 2 & 4 & \\
\hline 41 - 45 años & 3 & 1 & 4 & \\
\hline 46 - 50 años & 2 & 0 & 2 & \\
\hline \multicolumn{5}{|l|}{ Vive con } \\
\hline Familiares & 3 & 0 & 3 & \multirow[t]{4}{*}{0.37} \\
\hline Independiente & 1 & 2 & 3 & \\
\hline Otro & 5 & 2 & 7 & \\
\hline Padres & 6 & 1 & 7 & \\
\hline \multicolumn{5}{|l|}{$\mathrm{N}^{\circ}$ hermanos } \\
\hline 1 hermano & 3 & 0 & 3 & \multirow[t]{5}{*}{0.93} \\
\hline 2 Hermanos & 1 & 1 & 2 & \\
\hline 3 Hermanos & 5 & 2 & 7 & \\
\hline 4 Hermanos & 3 & 1 & 4 & \\
\hline No Aplica & 3 & 1 & 4 & \\
\hline \multicolumn{5}{|c|}{ Nivel socioeconómico } \\
\hline Bajo & 1 & 0 & 1 & \multirow[t]{3}{*}{0.65} \\
\hline Medio & 10 & 5 & 15 & \\
\hline Medio Bajo & 4 & 0 & 4 & \\
\hline
\end{tabular}

* prueba exacta de Fisher

Fuente. Elaboración propia resultados estudio

La variabilidad total explicitada por las variables género, número de hermanos, con quien vive y resiliencia fue del $88 \%$. El primer eje mostró un $73.7 \%$ de la varianza total, y discriminó los cuidadores según con quien vive (Independiente vs con familiares). El segundo eje señaló un $14.3 \%$ sobre la variable resiliencia; además, mostró una leve relación con la variable género. No obstante, en esta última variable hay que tener en cuenta que solo un cuidador fue hombre y las demás mujeres (Figura 1).

Igualmente, se puede observar que aquellos con Alto nivel de resiliencia son cuidadores independientes; mientras que los que tienen un nivel bajo o moderado viven con un mayor número de hermanos $(>=2$ hermanos), con los padres o con familiares. 
Figura 1. Gráfico simétrico de Correspondencias Múltiple de Resiliencia

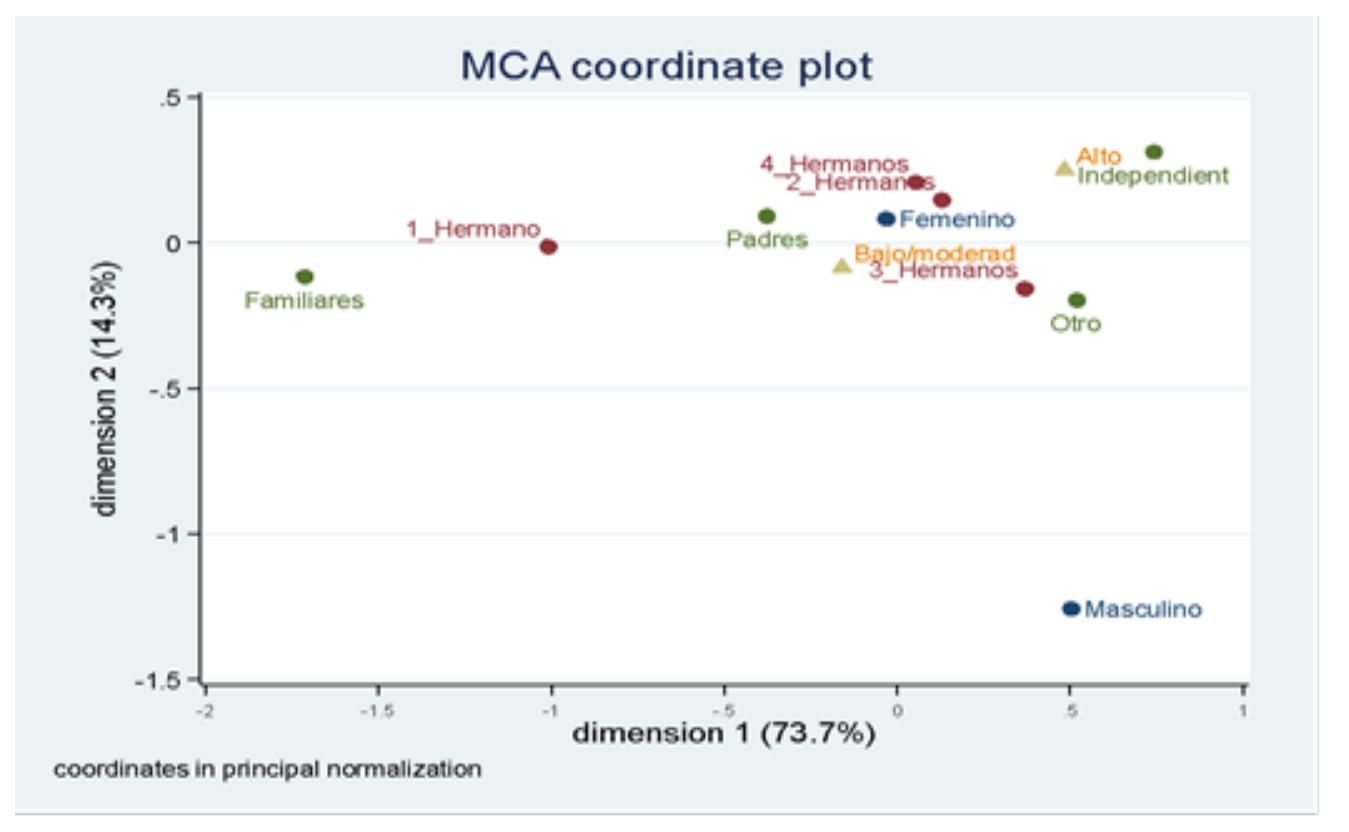

Fuente: Elaboración propia resultados estudio

\section{Discusión}

El objetivo de este estudio fue determinar la carga del cuidador y resiliencia de los enfermeros que brindan cuidado en una institución de atención domiciliaria en época de pandemia. Teniendo en cuenta lo planteado se utilizaron: una ficha para recolección de datos sociodemográficos, la Escala de Resiliencia en español y el cuestionario de sobrecarga del cuidador de Zarit. Estos instrumentos permitieron identificar las características que se reflejan en los resultados que se obtuvieron en el trabajo y que guardan semejanza con estudios similares.

En la atención domiciliaria el profesional de enfermería es capaz de fomentar y desarrollar una relación de ayuda, basada principalmente en la confianza, para llevar a cabo de una forma eficaz las intervenciones en salud. Por otro lado, trata de construir una relación terapéutica colaborativa, implicándose en la atención del paciente y del cuidador, y de este modo permitir una adecuación para su mejoría o mantenimiento efectivo (26).

Los resultados respecto a las características sociodemográficas del cuidador se asemejan a diversos estudios en donde se demuestra que la mayoría son mujeres quienes proporcionan el cuidado, con edades que oscilaron entre 22 y 38; de igual forma sufrieron de ansiedad, miedo e incertidumbre relacionados con la pandemia, desde las perspectivas del trabajo, el hogar, la familia y la comunidad (27).

Hay que mencionar que el estrés y la ansiedad son comunes en situaciones que generan incertidumbre como la pandemia que enfrentamos; circunstancias que inciden directamente en el personal de salud que debe enfrentar los servicios de hospitalización murales y domiciliarios (28). Los principales factores estresantes relacionados con el trabajo incluyen: inspecciones y exámenes frecuentes; una gran carga de trabajo; horas extraordinarias obligatorias (29); cuidado de adultos mayores (30); preocupaciones por las familias y la propia seguridad (31).

Otro rasgo que se observa tiene que ver con la sobrecarga intensa del personal de enfermería, asociada a quienes tienen: un menor número de hermanos (9), que viven con sus padres o familiares, aunque no hay posibles relaciones, además deben desempeñar otros roles al tener una unión o compañero, situaciones que, según lo evidencia la literatura, podrían incrementar la sobrecarga en el cuidado dado al estrés (27), convirtiéndose en una variable predictora de sobrecarga, tal como lo demuestra el estudio de Manso (32). Mientras que los que no presentan sobrecarga viven de manera independiente.

Al comparar las variables sociodemográficas con los 
resultados de la resiliencia, estadísticamente no se observaron diferencias significativas entre los que tenían el nivel de resiliencia bajo o moderado, y aquellos que mostraron una alta resiliencia. Este hallazgo es similar al encontrado por Fajardo en su investigación, en donde la población estaba conformada por cuidadores formales, conformado en su mayoría por mujeres con edades similares, quienes también presentaron asociación significativa entre el tipo de cuidador y el nivel de resiliencia (33).

Se puede indicar que en época de pandemia la resiliencia en el personal de salud especialmente en enfermería, permite inferir un sin número de reflexiones, dentro de las cuales se encuentran que al experimentar la pandemia algunas personas entraron en pánico, otras la minimizan o la niegan, mientras que otras culpan a algo o alguien. En definitiva, una perspectiva sana no niega los tiempos duros en la vida (34). Por tanto, las historias de aprendizaje y esperanza permiten valorar las estrategias positivas de afrontamiento en los enfermeros en cualquier nivel de atención en salud (35).

\section{Conclusiones}

- Dentro de las características sociodemográficas del personal de la enfermería de atención domiciliaria predomina el adulto joven de género femenino.

- La carga de cuidador a la que deben exponerse los profesionales de la enfermería al prestar los servicios domiciliarios y al intensificarse en tiempos de pandemia demuestran la capacidad de adaptación de la mayoría de cuidadores formales, que tienen experiencia superior a un año en estas labores.

- El nivel de resiliencia y sobrecarga del cuidador de los enfermeros de atención domiciliaria se presentan de manera indiferenciada.

- De la misma forma, la conformación del núcleo familiar de estos profesionales de la salud es un indicador importante para medir la resiliencia, pues se evidencia que los profesionales con mayor independencia se adaptan con más facilidad a cambios tan repentinos, frente a quienes no la tienen. Estos factores determinan que del aumento de la responsabilidad en los entornos familiares y el velar por la salud no solo de las personas que se cuidan sino también de los más cercanos al entorno familiar, hace que los enfermeros fomenten estrategias de cuidado, a través de las cuales el aislamiento y las medidas de bioseguridad son el soporte de la atención.

\section{Limitaciones}

Al momento de realizar la discusión sobre el tema estudiado, hay carencia de literatura, especialmente en todo lo que tiene que ver con la atención domiciliaria y la pandemia de Covid-19. Por tal motivo el tamaño de la muestra y el muestreo por conveniencia, no permiten establecer medidas de asociación.

\section{Conflicto de Intereses}

Los autores declaran no tener ningún conflicto de interés.

\section{Referencias Bibliograficas}

1. Navarro-Abal Y, López-López MJ, Climent-Rodríguez JA, Gómez-Salgado J. Sobrecarga, empatía y resiliencia en cuidadores de personas dependientes. Gac Sanit. 2019;33(3):268-71.

2. Becoña E. Resiliencia: Definicion, caracteristicas y utilidad del concepto. Rev Psicopatol y Psicol Clin. 2006;11(3):125-46.

3. Quiceno JM, Vinaccia S. Resiliencia, peccepción de enfermedad, creenciay afrontamiento espiritual reliigioso y calidad de vida relacionada con la salud en pacientes con diagnóstico de artritis reumatoide. Psicol Caribe. 2013;30(3):590-619.

4. Uriarte Arcineaga JDD. La resiliencia. Una nueva perspectiva en psicopatología del desarrollo Resilience. A new perspective into developmental psychopathology. Rev Psicodidáctica [Internet]. 2005;10(2):61-79. Available from: https://www.redalyc.org/pdf/175/17510206.pdf

5. Ospina Muñoz DE. La medición de la resiliencia. Investig y Educ en Enfermería. 2007;25:58-65.

6. Arrogante O. Resiliencia en Enfermería: definición, evidencia empírica e intervenciones. Index Enferm [Internet]. 2015;24(4). Available from: https://dx.doi.org/10.4321/S1132-12962015000300009 
7. wagnild G, young H. Development and Psychometric Evaluation of the Resilience Scale. J Nurs Meas. 1993;1(2):165-78.

8. Trigueros R, Álvarez JF, Aguilar-Parra JM, Alcaráz M, Rosado A. Validación y adaptación española de la escala de resiliencia en el contexto deportivo (ERCD). Psychol Soc Educ. 2017;9(2):311-24.

9. Caro Alonso P, Rodríguez-Martín B. Potencialidades de la resiliencia para los profesionales de Enfermería. Index Enferm. 2018;27(1-2).

10. Navarro-Abal Y, López-López MJ, Climent-Rodríguez JA. Engagement, resilience and empathy in nursing assistants. Enfermería Clínica [Internet]. 2018;28(2):103-10. Available from: https://doi.org/10.1016/j.enfcle.2017.08.012

11. Kunkle R, Chaperon C, Berger AM. Formal Caregiver Burden in Nursing Homes: An Integrative Review. West J Nurs Res. 2021;43(9):877-93.

12. Osorio RVB, Coral R del CI, Campos MS, Solarte KG, Ortiz VT. Cuidadores y cuidadoras familiares de personas con enfermedad crónica en Colombia: Más similitudes que diferencias. Salud Uninorte. 2015;31(2):25565 .

13. Crespo M, Rivas MT. La evaluación de la carga del cuidador: una revisión más allá de la escala de Zarit. Clin y Salud. 2015;26(1):9-16.

14. Song JA, Oh Y. The Association Between the Burden on Formal Caregivers and Behavioral and Psychological Symptoms of Dementia (BPSD) in Korean Elderly in Nursing Homes. Arch Psychiatr Nurs [Internet]. 2015;29(5):346-54. Available from: http://dx.doi.org/10.1016/j.apnu.2015.06.004

15. Zhang Y, Punnett L, Nannini A. Work-Family Conflict, Sleep, and Mental Health of Nursing Assistants Working in Nursing Homes. Work Heal Saf. 2017;65(7):295-303.

16. Crespo M, Fernández-Lansac V. Resiliencia en cuidadores familiares de personas mayores dependientes. An Psicol. 2015;31(1):19-27.

17. Muñoz Sánchez J. Abordaje de la resiliencia desde el proceso de atención de enfermería: a propósito de un caso. Rev Enfermería y Salud Ment. 2021;(17):22-6.

18. Alvarez Y, Delgado A, Madrigal D. Vulnerabilidad Y Resiliencia De Las Cuidadoras Familiares En Medio De La Pandemia De La Covid- Vulnerability and Resilience of Family Caregivers in the Midst of the Covid-19 Pandemic in the Municipality of Guisa, Province of Granma, Republic of Cuba. Rev Cient retos la Ccencia. 2020;4(2):1-14.

19. Lacerda MR, Tonin L, Hermann AP, Caceres NT de G. Recomendações Em Tempos De Covid-19: Um Olhar Para O Cuidado Domiciliar. Rev Bras Enferm [Internet]. 2020;73(2):1-13. Available from: https://www.

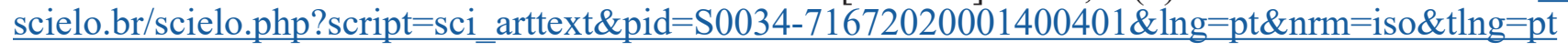

20. Mejías Estévez MJ. Primary and home care at the end of life in the COVID-19's time. Semergen. 2020;46(8):507-9.

21. Adams JG, Walls RM. Supporting the Health Care Workforce during the COVID-19 Global Epidemic. JAMA - J Am Med Assoc. 2020;323(15):1439-40.

22. Soto-Rubio A, Giménez-Espert MDC, Prado-Gascó V. Effect of emotional intelligence and psychosocial risks on burnout, job satisfaction, and nurses' health during the covid-19 pandemic. Int J Environ Res Public Health. 2020;17(21):1-14.

23. Heilemann M, Lee K, Kury F. The resilience scale. J Nurs Meas. 2003;11(1):61-72.

24. Vélez Lopera JM, Berbesí Fernández D, Cardona Arango D, Segura Cardona A, Ordóñez Molina J. Validación de escalas abreviadas de zarit para la medición de síndrome del cuidador primario del adulto mayor en Medellín. Aten Primaria. 2012;44(7):411-6.

25. Ministerio de Salud de Colombia. Resolución 8430 DE 1993. Colombia; 1993 p. 1-19.

26. Margañon SA, Rodriguez N. Sobrecarga Del Cuidador En Atención Domiciliaria. Horiz Sanit. 2018;17(3):179-87. 
Scientific Journal of Nursing

27. Varghese A, Kondaguli S V, Naser AY, Deepika C. Decline in the mental health of nurses across the globe during COVID-19 : A systematic review and meta-analysis. J Glob Health. 2021;11:1-15.

28. Odom-Forren J. Nursing Resilience in the World of COVID-19. J Perianesthesia Nurs [Internet]. 2020;35(6):555-6. Available from: https://doi.org/10.1016/j.jopan.2020.10.005

29. Leng M, Xiu H, Yu P, Feng J, Wei Y, Cui Y, et al. Current state and influencing factors of nurse resilience and perceived job-related stressors. J Contin Educ Nurs. 2021;51(3):132-7.

30. Ouslander JG, Grabowski DC. COVID-19 in Nursing Homes: Calming the Perfect Storm. J Am Geriatr Soc. 2020;68(10):2153-62.

31. Huang H, Xie Y, Chen Z, Xiao M, Cao S, Mi J, et al. Nursing Home in the COVID-19 Outbreak Challenge Recovery, and Resiliency. J Gerontol Soc Work [Internet]. 2020;00(00):1-5. Available from: https://doi.org/10.1 $\underline{080 / 01634372.2020 .1787576}$

32. Manso ME, Sanchez MDPS, Cuellar I. Health and perceived overload in people involved in family care in a rural area. Clin y Salud. 2013;24(1):37-45.

33. Ramos EF, Soto Morales AM, Henao Castaño AM. Sobrecarga del cuidador del adulto mayor en el barrio tierra firme de Ibagué ( Colombia ). SaludUninorte. 2020;35(2):264-76.

34. Wicks R. Resilience in a time of crisis. Nursing (Lond). 2020;49(4):1-2.

35. Thusini S. Critical care nursing during the COVID-19 pandemic: A story of resilience. Br J Nurs. 2020;29(21):1232-6. 